\title{
Identification of a cytosine methyltransferase that improves transformation efficiency in Methylomonas sp. DH-1
}

\author{
Jun Ren, Hyang-Mi Lee, Thi Duc Thai and Dokyun Na* (D)
}

\begin{abstract}
Background: Industrial biofuels and other value-added products can be produced from metabolically engineered microorganisms. Methylomonas sp. DH-1 is a candidate platform for bioconversion that uses methane as a carbon source. Although several genetic engineering techniques have been developed to work with Methylomonas sp. DH-1, the genetic manipulation of plasmids remains difficult because of the restriction-modification (RM) system present in the bacteria. Therefore, the RM system in Methylomonas sp. DH-1 must be identified to improve the genetic engineering prospects of this microorganism.

Results: We identified a DNA methylation site, TGGCCA, and its corresponding cytosine methyltransferase for the first time in Methylomonas sp. DH-1 through whole-genome bisulfite sequencing. The methyltransferase was confirmed to methylate the fourth nucleotide of TGGCCA. In general, methylated plasmids exhibited better transformation efficiency under the protection of the RM system than non-methylated plasmids did. As expected, when we transformed Methylomonas sp. DH-1 with plasmid DNA harboring the psy gene, the metabolic flux towards carotenoid increased. The methyltransferase-treated plasmid exhibited an increase in transformation efficiency of $2.5 \times 10^{3} \mathrm{CFU} / \mathrm{\mu g}(124 \%)$. The introduced gene increased the production of carotenoid by $26 \%$. In addition, the methyltransferase-treated plasmid harboring anti-psy sRNA gene exhibited an increase in transformation efficiency by $70 \%$ as well. The production of carotenoid was decreased by $40 \%$ when the psy gene was translationally repressed by anti-psy sRNA.

Conclusions: Plasmid DNA methylated by the discovered cytosine methyltransferase from Methylomonas sp. DH-1 had a higher transformation efficiency than non-treated plasmid DNA. The RM system identified in this study may facilitate the plasmid-based genetic manipulation of methanotrophs.
\end{abstract}

Keywords: Methylomonas sp. DH-1, Transformation efficiency, DNA methylation, Cytosine methyltransferase

\section{Background}

Although methane contributes to the greenhouse effect much more than carbon dioxide does, it is a useful feedstock for methanotrophs, which are bacteria that utilize methane as a carbon source [1-3]. Methane can be converted into methanol [4], and methanol can be metabolized to many other value-added chemicals such

\footnotetext{
*Correspondence: blisszen@cau.ac.kr
}

Department of Biomedical Engineering, Chung-Ang University,

Seoul 06974, Republic of Korea as L-glutamate [5, 6], L-lysine [7, 8], cadaverine [9, 10], $\alpha$-humulene [11], mesaconate, and (2S)-methyl-succinate [12] in metabolically engineered methanotrophs [13].

For efficient genetic engineering, genetic manipulation tools have been developed to work with methanotrophs [14-17]. Recently, the type l Methylomonas sp. DH-1 was isolated from brewery waste sludge, and several engineering tools have been developed [18]. This bacterium has been favored in diverse examples of metabolic engineering: the conversion of methane to methanol [18] and the production of value-added 
chemicals such as acetone $[19,20]$, succinate [21], and D-lactate [22].

The first hurdle in genetic engineering is to develop an efficient transformation method. In prokaryotes, DNA methylation and degradation by restrictionmodification (RM) systems, which are rudimentary bacterial immune systems, are yet to be identified [23]. Usually, foreign DNA is not methylated and is thus destroyed by host restriction enzymes. The methylation of particular sequences in the host genome protects those sequences from cleavage by host restriction enzymes $[24,25]$. A previous study showed that $88 \%$ of bacterial genomes contain RM systems and that $44 \%$ of bacterial genomes carry four or more RM systems [26]. Recently, the process of DNA methylation is utilized for epigenetic regulation [27] and nanopore sequencing [28].

Although several genetic manipulation techniques have been developed to metabolically engineer Methylomonas sp. DH-1 [29], the low transformation efficiency due to the inherent RM system has been an obstacle. In this study, we aimed to identify the RM system in Methylomonas sp. DH-1 and use it for enhanced genetic manipulation with plasmid DNA. Discovering the RM system of Methylomonas sp. DH-1 would enable the establishment of transformation techniques for efficient genetic manipulation.

\section{Results and discussion}

Identification of Methylomonas sp. DH-1 methylation site

To identify the RM system, the genome of Methylomonas sp. DH-1 was analyzed by whole-genome bisulfite sequencing (WGBS). Interestingly, only the TGGCCA motif was identified (Fig. 1a). In the REBASE database [30, 31], Methylomonas sp. DH-1 contains 12 RM systems in its genome and two in its native plasmid (Fig. 1b). According to REBASE, it was predicted that the cytosine methyltransferase AYM39_01025 (Additional file 1) would recognize the GGCC sequence for methylation, which is similar to the identified methylation site TGGCCA, in which the fourth nucleotide $(C)$ was methylated in our results. Therefore, this cytosine methyltransferase was selected as a potential methylase for TGGCCA.

\section{Digestion protection assay}

To investigate whether the selected cytosine methyltransferase (AYM39_01025) was able to recognize the identified sequence (TGGCCA) instead of the predicted sequence (GGCC), we conducted a DNA protection assay against digestion, using several restriction enzymes. When the cytosine methyltransferase protein was overexpressed in E. coli BL21 (DE3), the protein formed an inclusion body even though it was co-expressed with chaperones (pGro7 and pTf16). Thus, we could not perform the in vitro assay requiring purified methyltransferase. Instead, we introduced a plasmid harboring the

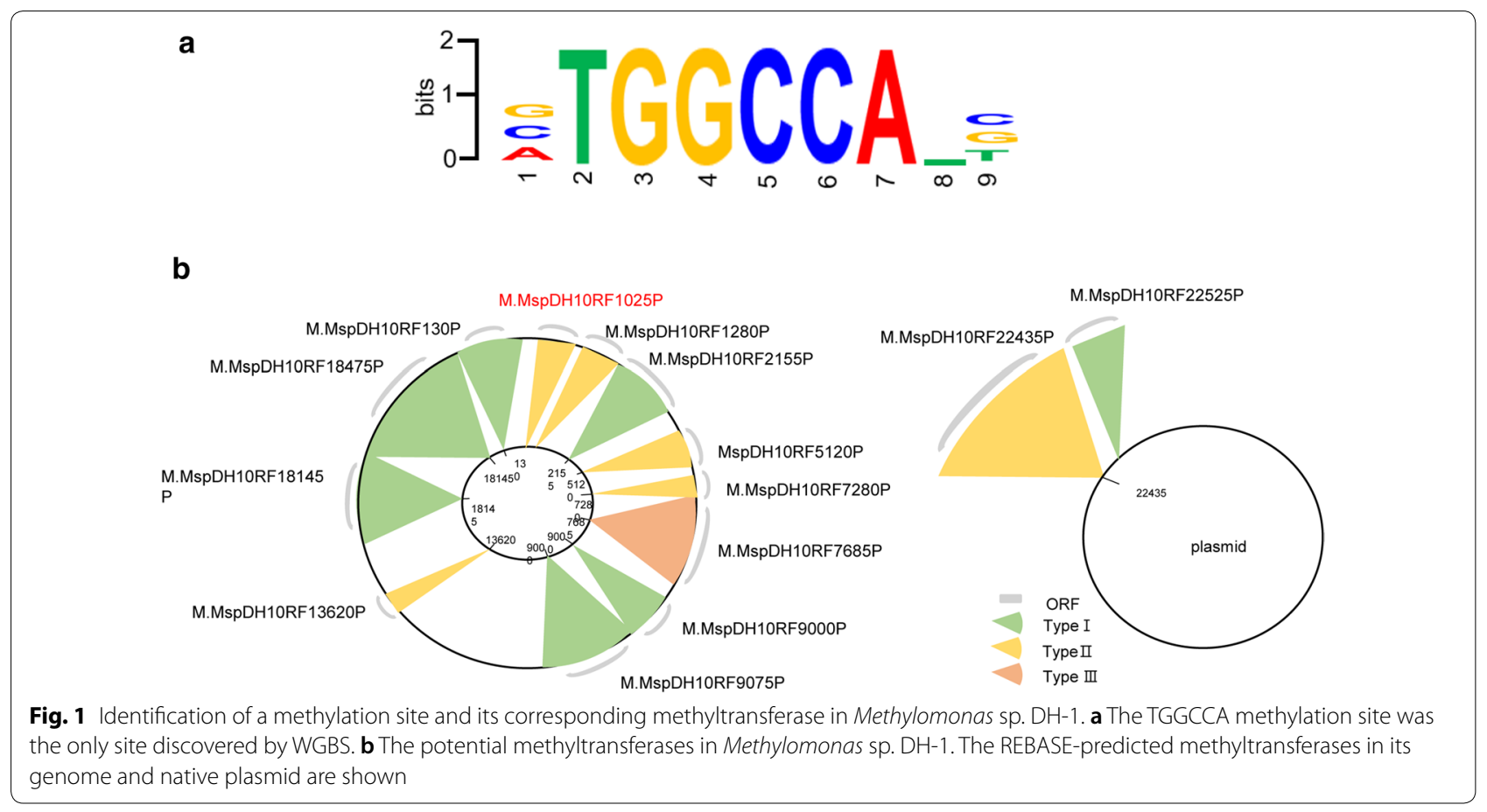


methyltransferase gene and TGGCCA sites into the $E$. coli JM110 strain (dam and $d c m$ methylase genes were deleted). Since the cytosine methyltransferase was under the control of the T5 promoter with a lac operator, we could obtain a non-methylated or methylated plasmid by IPTG. For further analysis, the plasmid was extracted from $E$. coli JM110 strain.

According to the REBASE annotations, the cytosine methyltransferase of Methylomonas sp. DH-1 was predicted to methylate the GGCC sequence, while the only methylation site identified in Methylomonas sp. DH-1 by WGBS was TGGCCA. To confirm that the cytosine methyltransferase recognized TGGCCA instead of GGCC, several restriction enzymes that contain GGCC in their restriction sites were used: $M s c \mathrm{I}$ (TGGCCA), ApaI (GGGCCC), and NotI (GCGGCCGC). We also used EcoRl (GAATTC) and Xbal (TCTAGA) restriction enzymes as negative controls. The plasmid harboring the cytosine methyltransferase gene contained all of the above-mentioned restriction sites, as well. If the methylation site was GGCC, the restriction enzymes (MscI, ApaI, and NotI) would not be able to cleave the plasmid DNA. As shown in Fig. 2a, most restriction enzymes were able to cleave both the non-methylated and methylated plasmids, but $M s c$ I failed to cleave the methylated plasmid. This result indicated that the cytosine methyltransferase recognized TGGCCA and not GGCC.

To identify the cytosine nucleotide methylated by the cytosine methyltransferase, the methylated plasmid was analyzed by bisulfite sequencing. In bisulfite sequencing, only non-methylated cytosines are converted to uracil, and during PCR, the uracil is converted to T. Methylated cytosines are not changed by bisulfite sequencing. As shown in Fig. 2b, TGGCCA in the non-methylated plasmid was converted to TGGTTA, indicating that the cytosines were non-methylated, as expected. In the methylated plasmid, only the fifth cytosine in TGGCCA was converted to $\mathrm{T}$, indicating that the fourth cytosine was methylated by the cytosine methyltransferase.

\section{Methylation of plasmid DNA increased transformation efficiency}

The plasmid harboring the psy (phytoene synthase) gene was constructed (Fig. 3a and Additional file 2) and cotransformed into $E$. coli JM110 with the plasmid harboring the cytosine methyltransferase gene. The psy gene is involved in the biosynthetic pathway that produces carotenoids. For the methylation of the plasmid containing $p s y$, the media were supplemented with $0.1 \mathrm{mM}$ IPTG to induce the expression of the cytosine methyltransferase. Since E. coli contains two plasmids (psy plasmid + cytosine methyltransferase plasmid), the plasmids were separated by gel electrophoresis, and the psy plasmid was

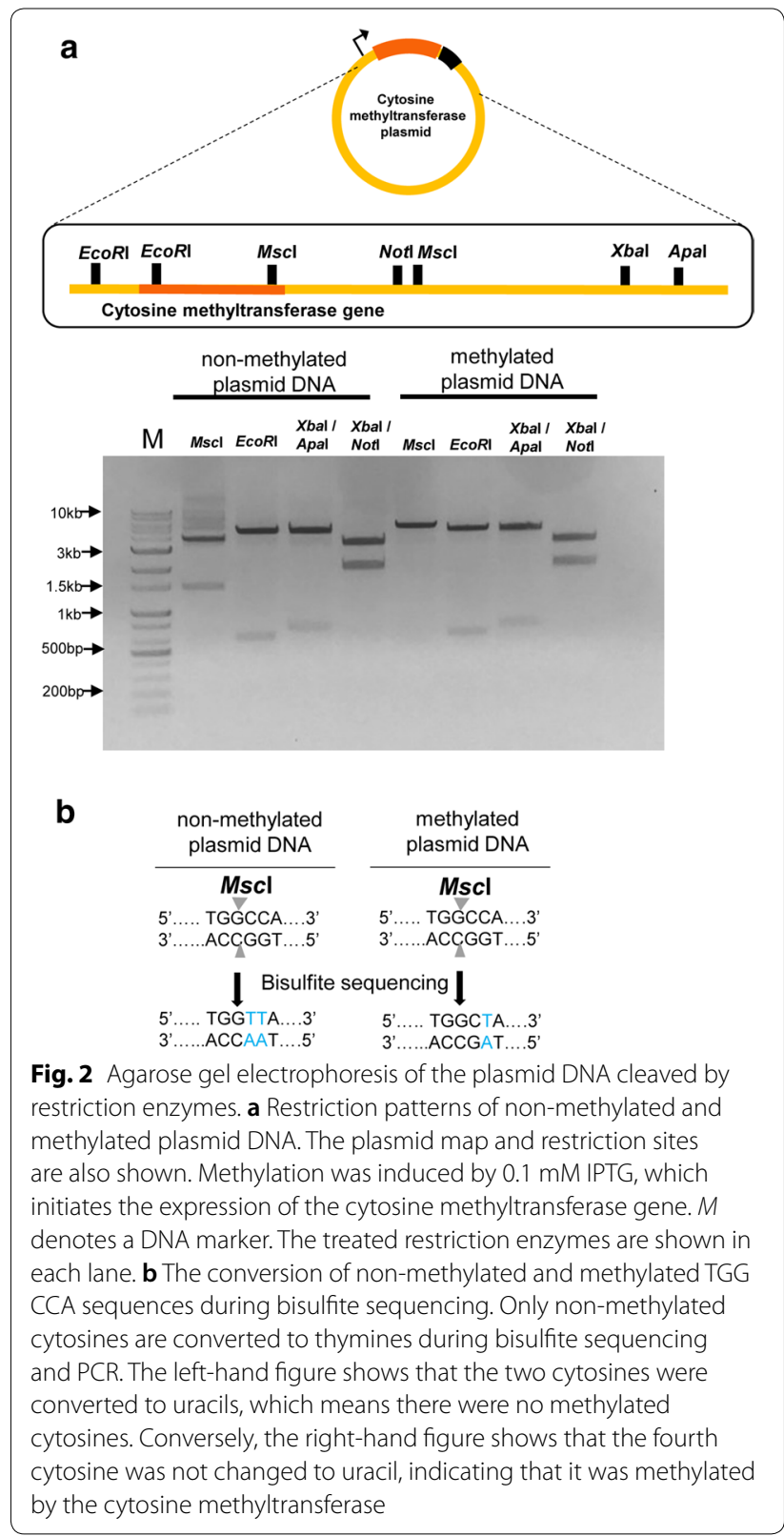

extracted from the gel (Fig. 3b). The non-methylated plasmid was also extracted from the cell without IPTG to create a control sample in which the expression of the cytosine methyltransferase was not induced.

The extracted plasmids were transformed into Methylomonas sp. DH-1 by electroporation. Since there are no artificial plasmids that exist separate from the genome of Methylomonas sp. DH-1, we measured the genome integration efficiencies of the psy gene involved in the carotenoid biosynthetic pathway (Fig. 4a) to deduce the transformation efficiency. The transformation efficiency of the methylated DNA of the psy plasmid was 


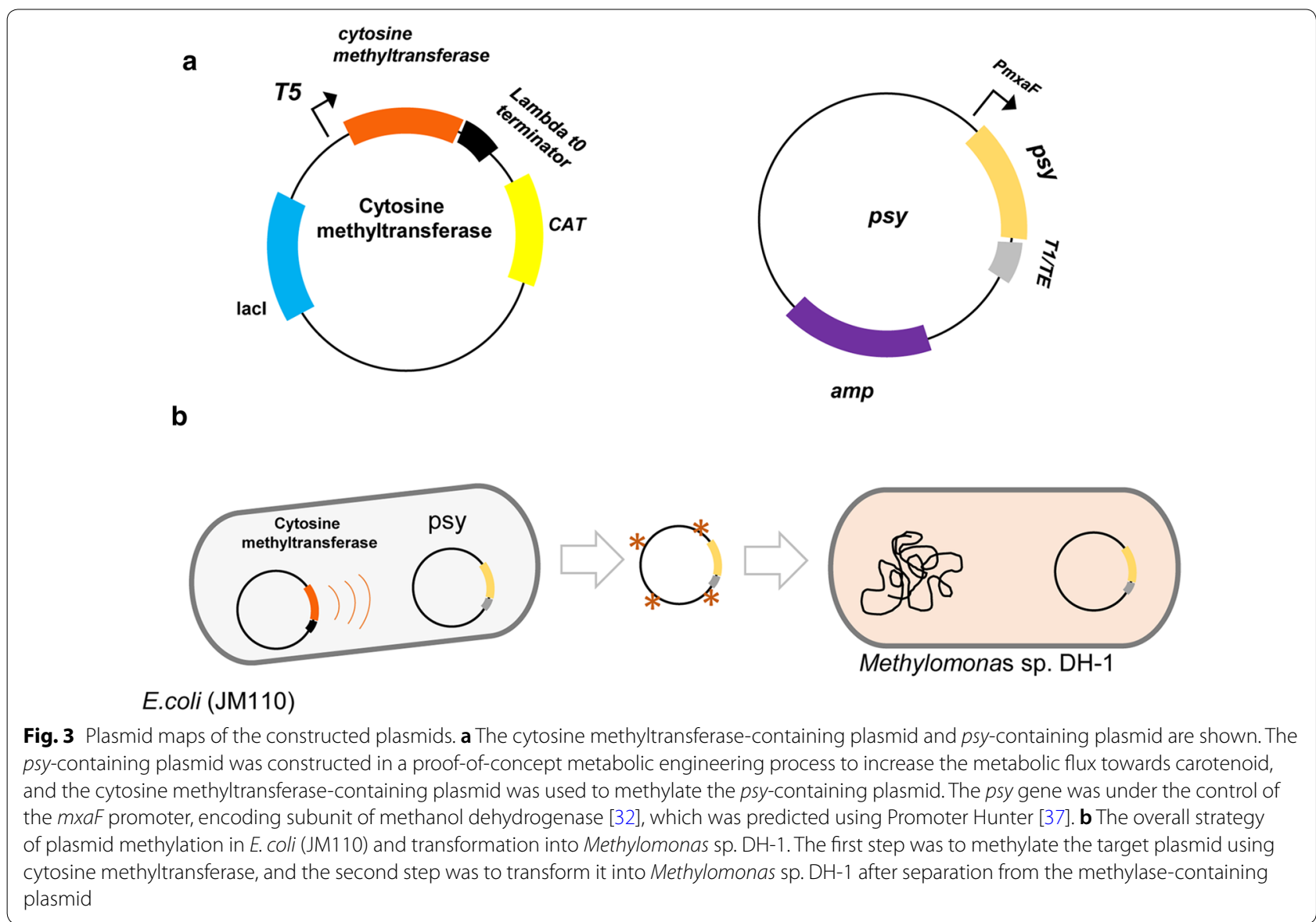

$2.5 \times 10^{3} \mathrm{CFU} / \mu \mathrm{g}$ of DNA. The efficiency was increased by $124 \%$ compared with that of the non-methylated plasmid DNA (Fig. 4b). Despite of methylation, the efficiency increase was not dramatic. We think that the introduced plasmids were easily integrated into the genome of Methylomonas sp. DH-1 by recombinases and thus the protection of plasmids by methylation was not essential in genome integration experiments. However, to date there are no artificial plasmids available for Methylomonas sp. DH-1, which is independent from its genome. For the development of artificial plasmids, the protection of plasmids by methylation is essential and the identification of a cytosine methylation system is the first step for the development. In this regard, the newly identified methylation system would facilitate the development of artificial plasmids as well as other biotechnological techniques based on plasmids.

For further evaluation of methylation effect on transformation, we removed the three methylation sites in the psy plasmid by mutating nucleotides: one in an intergenic region and two in the coding region of $p s y$. The former site was converted from TGGCCA to TGT $\mathrm{CCA}$, and the latter two were converted from GTG

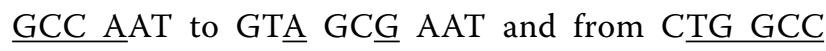
AAA to CTA GCG AAA based on codon degeneracy not to mutate amino acids (Additional file 3: Fig S1a). As shown in Additional file 3: Fig. S1b, the deletion of the methylation sites increased transformation efficiency similar to that of the methylated psy plasmid. This indicates that methylation of plasmid DNA by the identified cytosine methyltransferase protects plasmid DNAs from the RM system of Methylomonas sp. DH-1 and increases the transformation efficiency, which may facilitate the genetic manipulation of Methylomonas sp. DH-1.

To investigate the effects of plasmid size and methylation on transformation efficiency, we constructed three different plasmids with a different length $(5-7 \mathrm{~kb})$. As shown in Additional file 3: Fig. S2, methylation increased transformation efficiency while plasmid length did not show any significant effect on transformation efficiency. This indicates that transformation efficiency is dependent on methylation, not plasmid size. Furthermore, we measured the growths of non-transformed wild-type cells and transformed cells with methylated plasmids (the $p s y$ plasmid or anti-psy sRNA plasmid). As shown in Additional 


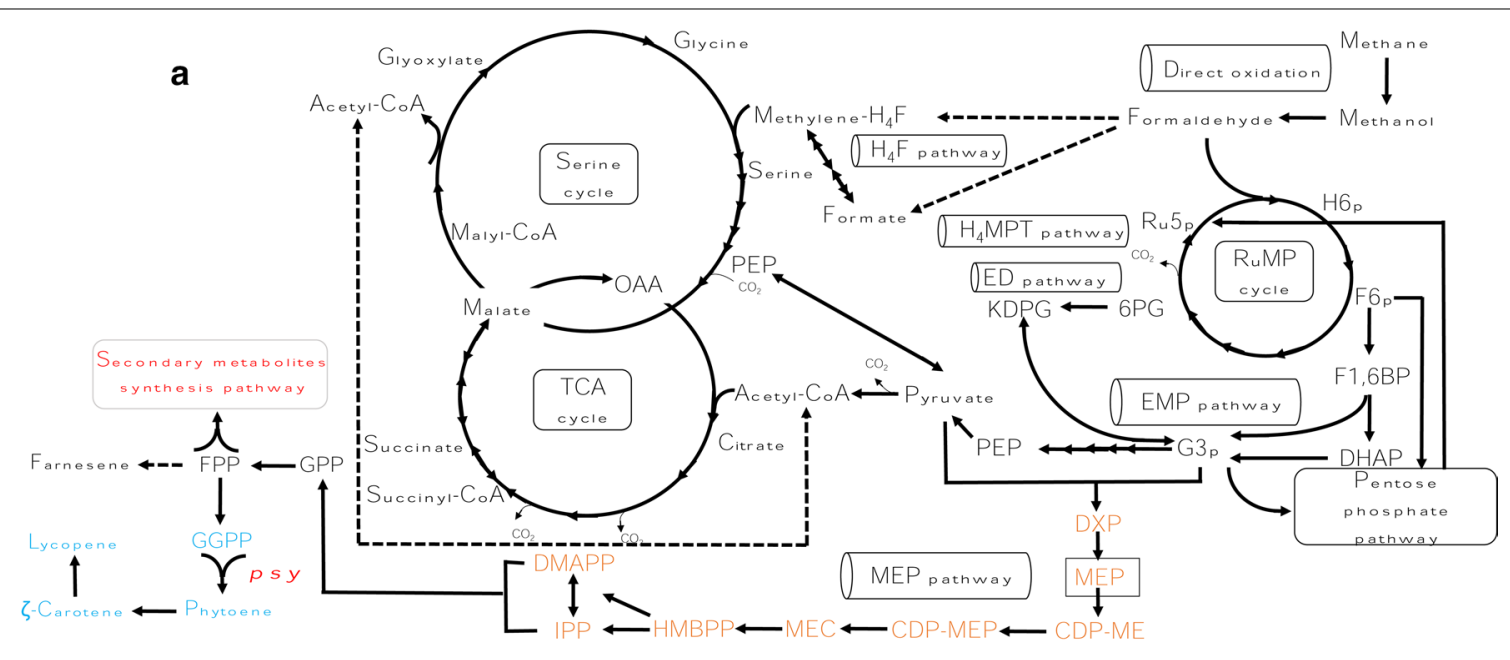

b

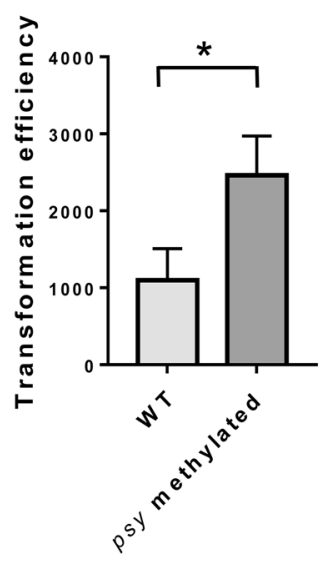

C

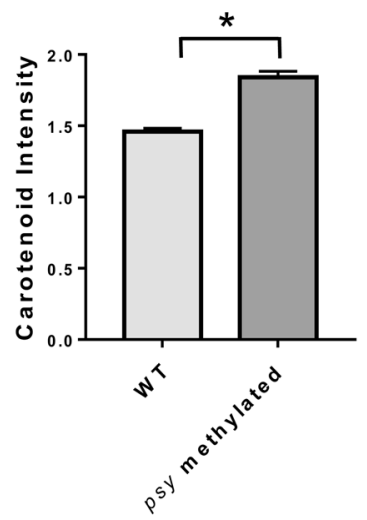

Fig. 4 The transformation efficiency of methylated plasmid DNAs. a The overall biosynthetic pathway towards carotenoid. The psy gene is indicated. b Transformation efficiencies of non-methylated plasmids (light gray bar) and methylated plasmids (dark gray bar) in Methylomonas sp. DH-1. The maps of the two plasmids are shown in Fig. 3a. Standard deviations were calculated from triplicates. The asterisk (*) denotes $p$ values $<0.05$. c Carotenoid intensity in Methylomonas sp. DH-1 cells after transformation with the methylated psy plasmid. The intensity was measured using multi-detection microplate reader, and the carotenoid intensity was obtained $8 \mathrm{~h}$ after cultivation. Standard deviations were calculated from triplicates

file 3: Fig. S3, their growth rates are very similar, showing that there could be no significant changes in cellular physiology.

Methylomonas sp. DH-1 carries a complete MEP pathway for carotenoid production [19]. The selected gene, $p s y$, is involved in the carotenoid biosynthetic pathway. The gene was designed to be expressed by the promoter of the mxaF gene [32] (Fig. 4a). When the plasmid containing the psy gene was introduced into Methylomonas sp. DH-1, the psy gene was integrated into the genome by homologous recombination. The additional copy of the psy gene increased carotenoid biosynthesis by $26 \%$ (Fig. 4c).

Synthetic sRNAs have been utilized to increase the production of desired substances by regulating gene expression [33]. Synthetic sRNAs were designed to bind to the nucleotides in the translation initiation regions of mRNAs, and thereby they repressed the translation of mRNAs by preventing the binding of ribosomes in assistance of Hfq protein [34]. In this study, we constructed a plasmid containing an anti- $p s y$ synthetic sRNA gene to investigate the methylation effect on transformation efficiency and also to investigate the knock-down effect of the psy gene on carotenoid production (Fig. $5 \mathrm{a}$ and Additional file 4).

Synthetic sRNAs are composed of two elements: a target binding region and a scaffold. In previous studies, various scaffolds originated from $E$. coli were used including MicC and SgrS [33, 35]. Of many inherent sRNA genes, RyhB was known to operate in the 


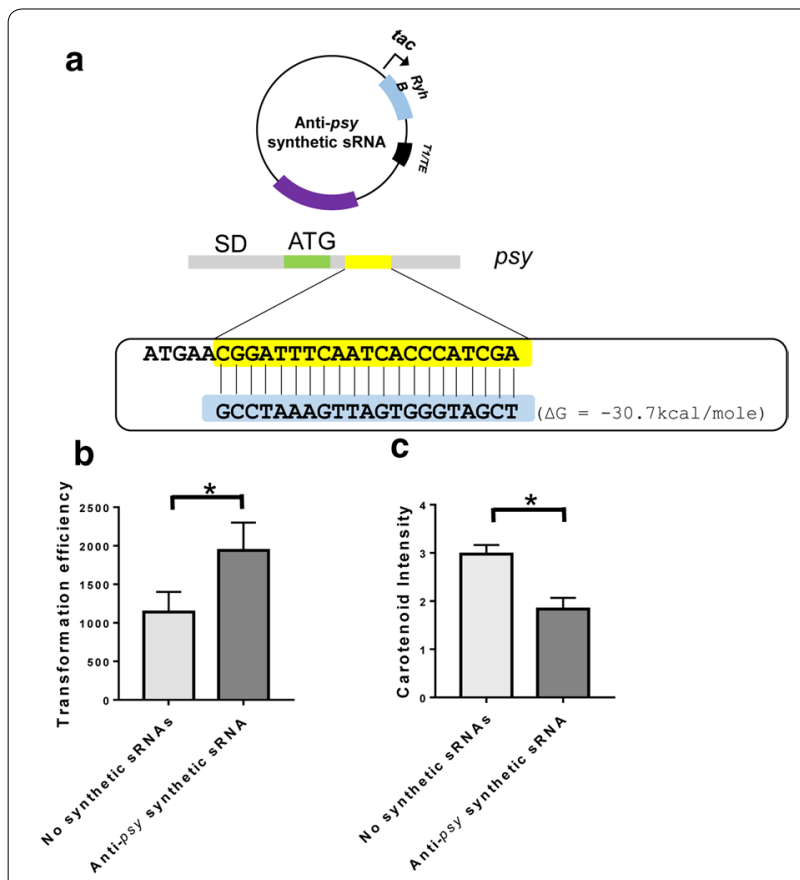

Fig. 5 Transformation efficiency of a methylated plasmid harboring anti-psy synthetic sRNA gene. a An anti-psy synthetic sRNA gene was designed to reduce the expression of the psy gene. The synthetic sRNA binds to the coding region of the psy gene and represses the translation of the psy mRNA. The binding energy was calculated by mfold [38]. b Transformation efficiencies of the non-methylated plasmid (light gray bar) and methylated plasmid (dark gray bar) in Methylomonas sp. DH-1. Standard deviations were calculated from triplicates. The asterisk ${ }^{*}$ ) denotes $p$ values $<0.05$. c Carotenoid intensity (arbitrary unit) in Methylomonas sp. DH-1 cells. The intensity was measured using multi-detection microplate reader, and the carotenoid intensity was obtained $8 \mathrm{~h}$ after cultivation. Standard deviations were calculated from triplicates. The asterisk $(*)$ denotes $p$ values $<0.05$ absence of Hfq protein [36] and thus was expected to work in various bacterial species. We designed an antipsy synthetic sRNA using the scaffold of RyhB sRNA. The anti-psy synthetic sRNA gene was under the control of tac promoter.

When the plasmid harboring the anti-psy synthetic sRNA gene was transformed after methylation, its transformation efficiency was enhanced by $70 \%$ compared with the non-methylated plasmid (Fig. 5b). In addition, when the synthetic sRNA gene was integrated into the genome of Methylomonas sp. DH-1, the synthetic sRNA decreased carotenoid production by $40 \%$ compared with that of wild-type Methylomonas sp. DH-1 (Fig. 5c). These results indicate that methylation of plasmids can improve transformation efficiency as well as that synthetic sRNAs based on a
RyhB scaffold can be used to regulate the expression of genes in Methylomonas sp. DH-1.

\section{Conclusions}

In this study, we identified a novel cytosine methyltransferase and its methylation site for the first time in Methylomonas sp. DH-1. The methylase was utilized to increase transformation efficiency by protecting plasmid DNAs from the RM system of Methylomonas sp. DH-1. Transformation is the first barrier in the genetic manipulation of bacteria, and with the aid of the methylase, the transformation barrier was effectively overcome. The use of the methylase for methylating insertional genes may facilitate the metabolic engineering of value-added products in Methylomonas sp. DH-1.

\section{Methods}

Strains, antibiotics, primers, and culture conditions

The E. coli DH5 $\alpha$ strain was used for gene cloning and plasmid preparation, and the E. coli JM110 strain, traD36 lacl $^{q} \Delta$ (lacZ) M15 proA $^{+} B^{+}$IrpsL (Str') thr leu thi lacY galK galT ara fhuA dam dcm glnV44 $\triangle($ lac-proAB) was used for plasmid methylation. E. coli cells were cultured in Luria-Bertani (LB) broth (1\% tryptone, $0.5 \%$ yeast extract, and $1 \% \mathrm{NaCl})$ or on LB plates $(1.5 \% \mathrm{w} / \mathrm{v}$ agar) at $37{ }^{\circ} \mathrm{C}$ in the presence of appropriate antibiotics $(25 \mu \mathrm{g} /$ $\mathrm{mL}$ of chloramphenicol and/or $10 \mu \mathrm{g} / \mathrm{mL}$ of Ampicillin). A cytosine methyltransferase (Additional file 1) expression vector was then constructed, and its expression was regulated by IPTG. For methylation, $0.1 \mathrm{mM}$ IPTG was used. Next, the $p s y$ plasmid was constructed.

Methylomonas sp. DH-1 was cultured in a nitrate mineral salt (NMS) medium containing $10 \mu \mathrm{M} \mathrm{CuCl}_{2} \cdot 2 \mathrm{H}_{2} \mathrm{O}$ as described previously [18]. Methylomonas sp. DH-1 cells were cultured in a $500 \mathrm{~mL}$ baffled flask sealed with a screw cap containing $100 \mathrm{~mL}$ of NMS medium at $30^{\circ} \mathrm{C}$ and $250 \mathrm{rpm}$. Methane was supplied to a final concentration of $30 \%(\mathrm{v} / \mathrm{v})$ by gas substitution using a gas-tight syringe, and the headspace was refreshed daily. During carotenoid intensity measurement, methanol $(0.1 \%)$ was used as a carbon source instead of methane because the Methylomonas sp. DH-1 cells were cultured in a 96-well plate.

\section{Whole-genome bisulfite sequencing and plasmid construction}

A whole-genome bisulfite sequencing of Methylomonas sp. DH-1 was carried out according to the manufacturer's instruction [39]. Briefly, genomic DNA of Methylomonas sp. DH-1 was extracted and was treated with bisulfite to convert unmethylated cytosines to uracils while retaining those which were methylated. Bisulfite-treated ssDNA 
fragments were randomly primed using a polymerase that can read uracil nucleotides to synthesize DNA strands containing a specific sequence tag. The $3^{\prime}$ ends of the newly synthesized DNA strands were then selectively tagged with a second sequence tag. This process generated di-tagged DNA strands with known tags at their $5^{\prime}$ and $3^{\prime}$ ends. The di-tagged DNA strands were enriched by PCR, resulting in dsDNA strands.

After sequencing, the raw sequence reads were filtered based on quality. Trimming process was done to eliminate adapter sequences and bases with low quality from each read using Trimmomatic program [40]. The bases with low quality or $\mathrm{N}$ bases less than quality 3 from the ends of reads were trimmed. Also, using a sliding window method, bases of reads that did not qualify for a 4-base wide sliding window, and the average quality per window below 15 were trimmed. Afterwards, reads with length shorter than $36 \mathrm{bp}$ were dropped to produce cleaned data. The trimmed reads were mapped to a reference genome (Methylomonas sp. DH-1, ASM164468v1) with BSMAP based on SOAP (Short Oligo Alignment Program), which is a short reads mapping program for bisulfite sequencing in DNA methylation study [41]. The nucleotides around methylated cytosines $(-10 \sim+10 \mathrm{nt})$ were extracted and consensus sequence motifs were identified using MEME [42]. As a result, one single motif was identified (TGGCCA). The raw data files obtained from bisulfite sequencing are available to download at http://ssbio.cau.ac.kr/public/DH-1_1.fastq.gz and http:// ssbio.cau.ac.kr/public/DH-1_2.fastq.gz.

\section{Methylomonas sp. DH-1 electroporation}

Methylomonas sp. DH-1 was grown in a nitrate mineral salt (NMS) plate containing $10 \mu \mathrm{M} \mathrm{CuCl} \mathrm{Cl}_{2} \cdot 2 \mathrm{H}_{2} \mathrm{O}$ as described previously [23]. Cells were collected from plates using a spreader and resuspended in distilled water (DW) to make $\mathrm{OD}_{600}=0.8 .10 \mathrm{~mL}$ of the resuspended cells were harvested by centrifugation at $5000 \mathrm{rpm}$ at $4{ }^{\circ} \mathrm{C}$ for $10 \mathrm{~min}$. The pellet was washed with $10 \mathrm{~mL}$ of DW, transferred to a $15 \mathrm{~mL}$ conical tube, and centrifuged again at $5000 \mathrm{rpm}$ at $4{ }^{\circ} \mathrm{C}$ for $10 \mathrm{~min}$. The resulting pellet was resuspended in $100 \mu \mathrm{L}$ of DW and placed on ice. Fifty microliters of the resuspended cells were gently mixed with plasmid $500 \mathrm{ng}$ of DNA $(3-5 \mu \mathrm{L})$, and the mixture was transferred to an ice-cold $1 \mathrm{~mm}$ micropulser electroporation cuvettes (Bio-Rad, Hercules, California 94547, United States). Electroporation was performed using a micropulser electroporator (Gene Pulser II System, Bio-Rad, Herules, California 94547, United States) at $25 \mu \mathrm{F}$ and $200 \Omega$. After electrical discharge, $1 \mathrm{~mL}$ of NMS medium was immediately added to the cells. The cells were transferred into a $250 \mathrm{~mL}$ serum bottle with $10 \mathrm{~mL}$ of NMS medium and then incubated with $0.02 \%$ methane gas. After incubation at $30^{\circ} \mathrm{C}$ for $4 \mathrm{~h}$, the cells were collected by centrifugation at $5000 \mathrm{rpm}$ for $10 \mathrm{~min}$ at $25^{\circ} \mathrm{C}$. The cells were resuspended with $1 \mathrm{~mL}$ of NMS medium and spread onto selective NMS plates.

\section{Carotenoid measurement}

Methylomonas sp. DH-1 transformed with the psy plasmid (Additional file 2) was cultured in NMS medium containing methanol $(0.1 \%)$ at $30{ }^{\circ} \mathrm{C}$ until the stationary phase was reached. The Methylomonas sp. DH-1 was transferred to a 96-well plate containing $200 \mu \mathrm{L}$ of NMS medium and grown in a shaking format at $30{ }^{\circ} \mathrm{C}$. The $\mathrm{OD}_{450}$ absorbance at $8 \mathrm{~h}$ was measured to infer the relative carotenoid concentration using a multi-detection microplate reader (SpectraMax M2, Molecular Devices, Sunnyvale, CA, USA).

\section{Supplementary Information}

The online version contains supplementary material available at https://doi. org/10.1186/s13068-020-01846-1.

Additional file 1. Cytosine methyltransferase gene sequence in SnapGene file format.

Additional file 2. Psy plasmid map in SnapGene file format.

Additional file 3: Fig. S1. Transformation efficiency of the plasmid DNA in which methylation sites were changed to non-methylation sites. Fig. S2. The effects of plasmid length and methylation on transformation efficiency. Fig. S3. The growth curves of Methylomonas sp. DH-1 cells.

Additional file 4. Anti-psy sRNA plasmid map in SnapGene file format.

\section{Abbreviations}

RM: Restriction modification; psy: Phytoene synthase; NMS: Nitrate mineral salt; DW: Distilled water; WGBS: Whole-genome bisulfite sequencing; IPTG: Isopropyl $\beta$-D-1-thiogalactopyranoside; sRNA: Regulatory small RNA; TIR: Translation initiation region.

\section{Acknowledgements}

Not applicable.

\section{Authors' contributions}

DN supervised this study and revised the manuscript. JR carried out the bisulfite sequencing experiments and drafted the manuscript. HL performed the transformation of Methylomonas sp. DH-1. TD carried out the construction of the plasmids. All authors read and approved the final manuscript.

\section{Funding}

This research was supported by the C1 Gas Refinery Program through the National Research Foundation of Korea (NRF) funded by the Ministry of Science, ICT, and Future Planning (NRF-2016M3D3A1A01913244). This work was also supported by an NRF grant funded by the Korea government (MSIT; No. NRF-2018R1A5A1025077)

Availability of data and materials Not applicable.

Ethics approval and consent to participate Not applicable. 


\section{Consent for publication \\ Not applicable.}

\section{Competing interests}

The authors declare that they have no competing interests.

Received: 27 June 2020 Accepted: 30 November 2020 Published online: 07 December 2020

\section{References}

1. Clomburg JM, Crumbley AM, Gonzalez R. Industrial biomanufacturing: the future of chemical production. Science. 2017;355(6320):aag0804.

2. Hwang IY, Hur DH, Lee JH, Park CH, Chang IS, Lee JW, et al. Batch conversion of methane to methanol using Methylosinus trichosporium OB3b as biocatalyst. J Microbiol Biotechnol. 2015;25(3):375-80.

3. Kalyuzhnaya MG, Puri AW, Lidstrom ME. Metabolic engineering in methanotrophic bacteria. Metab Eng. 2015;29:142-52.

4. Xin JY, Cui JR, Niu JZ, Hua SF, Xia CG, Li SB, et al. Production of methanol from methane by methanotrophic bacteria. Biocatal Biotransform 2004:22(3):225-9.

5. Brautaset $T$, Jakobsen OM, Degnes KF, Netzer R, Naerdal I, Krog A, et al. Bacillus methanolicus pyruvate carboxylase and homoserine dehydrogenase I and II and their roles for L-lysine production from methanol at 50 degrees C. Appl Microbiol Biotechnol. 2010;87(3):951-64.

6. Brautaset T, Williams MD, Dillingham RD, Kaufmann C, Bennaars A, Crabbe

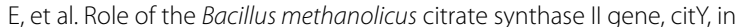
regulating the secretion of glutamate in L-lysine-secreting mutants. Appl Environ Microbiol. 2003;69(7):3986-95.

7. Naerdal I, Netzer R, Ellingsen TE, Brautaset T. Analysis and manipulation of aspartate pathway genes for L-lysine overproduction from methanol by Bacillus methanolicus. Appl Environ Microbiol. 2011;77(17):6020-6.

8. Jakobsen OM, Brautaset T, Degnes KF, Heggeset TM, Balzer S, Flickinger $M C$, et al. Overexpression of wild-type aspartokinase increases L-lysine production in the thermotolerant methylotrophic bacterium Bacillus methanolicus. Appl Environ Microbiol. 2009;75(3):652-61.

9. Naerdal I, Pfeifenschneider J, Brautaset T, Wendisch VF. Methanol-based cadaverine production by genetically engineered Bacillus methanolicus strains. Microb Biotechnol. 2015:8(2):342-50.

10. Irla M, Heggeset TM, Naerdal I, Paul L, Haugen T, Le SB, et al. Genomebased genetic tool development for Bacillus methanolicus: theta- and rolling circle-replicating plasmids for Inducible gene expression and application to methanol-based cadaverine production. Front Microbiol. 2016;7:1481.

11. Sonntag F, Kroner C, Lubuta P, Peyraud R, Horst A, Buchhaupt M, et al. Engineering Methylobacterium extorquens for de novo synthesis of the sesquiterpenoid alpha-humulene from methanol. Metab Eng. 2015:32:82-94

12. Sonntag F, Buchhaupt M, Schrader J. Thioesterases for ethylmalonylCoA pathway derived dicarboxylic acid production in Methylobacterium extorquens AM1. Appl Microbiol Biotechnol. 2014;98(10):4533-44.

13. Pfeifenschneider J, Brautaset T, Wendisch VF. Methanol as carbon substrate in the bio-economy: metabolic engineering of aerobic methylotrophic bacteria for production of value-added chemicals. Biofuel Bioprod Biorefin. 2017;11(4):719-31.

14. Puri AW, Owen S, Chu F, Chavkin T, Beck DA, Kalyuzhnaya MG, et al. Genetic tools for the industrially promising methanotroph Methylomicrobium buryatense. Appl Environ Microbiol. 2015:81(5):1775-81.

15. Yan X, Chu F, Puri AW, Fu Y, Lidstrom ME. Electroporation-based genetic manipulation in type I methanotrophs. Appl Environ Microbiol. 2016:82(7):2062-9.

16. Crombie A, Murrell JC. Development of a system for genetic manipulation of the facultative methanotroph Methylocella silvestris BI2. In: Methods in enzymology. 2011. pp. 119-33.

17. Ojala DS, Beck DAC, Kalyuzhnaya MG. Genetic systems for moderately halo (alkali)philic bacteria of the genus methylomicrobium. In: Methods in enzymology. 2011. pp. 99-118.

18. Hur DH, Na JG, Lee EY. Highly efficient bioconversion of methane to methanol using a novel type I Methylomonas sp DH-1 newly isolated from brewery waste sludge. J Chem Technol Biotechol. 2017;92(2):311-8.
19. Nguyen AD, Hwang IY, Lee OK, Hur DH, Jeon YC, Hadiyati S, et al. Functional analysis of Methylomonas sp DH-1 genome as a promising biocatalyst for bioconversion of methane to valuable chemicals. Catalysts. 2018;8(3):117

20. Hur DH, Nguyen TT, Kim D, Lee EY. Selective bio-oxidation of propane to acetone using methane-oxidizing Methylomonas sp. DH-1.J Ind Microbiol Biotechnol. 2017:44(7):1097-105.

21. Nguyen DTN, Lee OK, Hadiyati S, Affifah AN, Kim MS, Lee EY. Metabolic engineering of the type I methanotroph Methylomonas sp. DH-1 for production of succinate from methane. Metab Eng. 2019;54:170-9.

22. Lee JK, Kim S, Kim W, Kim S, Cha S, Moon H, et al. Efficient production of d-lactate from methane in a lactate-tolerant strain of Methylomonas sp. $\mathrm{DH}-1$ generated by adaptive laboratory evolution. Biotechnol Biofuels. 2019;12:234.

23. Bertani $G$, Weigle JJ. Host controlled variation in bacterial viruses. J Bacteriol. 1953;65(2):113-21.

24. Murray NE. Type I restriction systems: sophisticated molecular machines (a legacy of Bertani and Weigle). Microbiol Mol Biol Rev. 2000;64(2):412-34.

25. Hornby DP. DNA methyltransferases (EC 2.1.1.72 and EC 2.1.1.73). Methods Mol Biol. 1993;16:201-11.

26. Roberts RJ, Vincze T, Posfai J, Macelis D. REBASE—enzymes and genes for DNA restriction and modification. Nucleic Acids Res. 2007;35(Database issue):D269-70

27. Oliveira PH, Fang G. Conserved DNA methyltransferases: a window into fundamental mechanisms of epigenetic regulation in bacteria. Trends Microbiol. 2020

28. Tourancheau A, Mead EA, Zhang X-S, Fang GJb. Discovering and exploiting multiple types of DNA methylation from individual bacteria and microbiome using nanopore sequencing. 2020.

29. Ren J, Na D, Yoo SM. Optimization of chemico-physical transformation methods for various bacterial species using diverse chemical compounds and nanomaterials. J Biotechnol. 2018;288:55-60.

30. Roberts RJ, Vincze T, Posfai J, Macelis D. REBASE: restriction enzymes and methyltransferases. Nucleic Acids Res. 2003;31(1):418-20.

31. Roberts RJ, Vincze T, Posfai J, Macelis D. REBASE—restriction enzymes and DNA methyltransferases. Nucleic Acids Res. 2005;33(Database issue):D230-2.

32. Chistoserdova $L$, Kuhn M, Lidstrom ME. Identification of a promoter region for mxaF (moxF) from the type I methanotroph, Methylobacter albus BG8. FEMS Microbiol Lett. 1994;121(3):343-8.

33. Na D, Yoo SM, Chung $\mathrm{H}$, Park $\mathrm{H}$, Park JH, Lee SY. Metabolic engineering of Escherichia coli using synthetic small regulatory RNAs. Nat Biotechnol. 2013;31(2):170-4

34. De Lay N, Schu DJ, Gottesman S. Bacterial small RNA-based negative regulation: Hfq and its accomplices. J Biol Chem. 2013;288(12):7996-8003.

35. Noh M, Yoo SM, Yang D, Lee SY. Broad-spectrum gene repression using scaffold engineering of synthetic sRNAs. Acs Synth Biol. 2019:8(6):1452-61.

36. Hao Y, Zhang ZJ, Erickson DW, Huang M, Huang Y, Li J, et al. Quantifying the sequence-function relation in gene silencing by bacterial small RNAs. Proc Natl Acad Sci USA. 2011;108(30):12473-8.

37. Klucar L, Stano M, Hajduk M. phiSITE: database of gene regulation in bacteriophages. Nucleic Acids Res. 2010;38:D366-70.

38. Zuker M. Mfold web server for nucleic acid folding and hybridization prediction. Nucleic Acids Res. 2003;31(13):3406-15.

39. Nair SS, Luu PL, Qu WJ, Maddugoda M, Huschtscha L, Reddel R, et al. Guidelines for whole genome bisulphite sequencing of intact and FFPET DNA on the Illumina HiSeq X Ten. Epigenet Chromatin. 2018;11:24.

40. Bolger AM, Lohse M, Usadel B. Trimmomatic: a flexible trimmer for Illumina sequence data. Bioinformatics. 2014;30(15):2114-20.

41. Xi Y, Li W. BSMAP: whole genome bisulfite sequence MAPping program. BMC Bioinform. 2009;10:232

42. Bailey TL, Boden M, Buske FA, Frith M, Grant CE, Clementi L, et al. MEME suite: tools for motif discovery and searching. Nucleic Acids Res. 2009:37(suppl_2):W202-8.

\section{Publisher's Note}

Springer Nature remains neutral with regard to jurisdictional claims in published maps and institutional affiliations. 